\title{
A DISCRETE CURVE-SHORTENING EQUATION
}

\author{
Kazuaki Nakayama, Harvey Segur, and Miki Wadati \\ Dedicated to Martin D. Kruskal on the occasion of his 70th birthday.
}

\begin{abstract}
The usual "curve-shortening equation" describes the planar motion of a smooth curve that moves in a direction normal to itself with a speed proportional to its local curvature. We present here an analogous theory for the planar motion of a discrete (i.e., piecewise linear) curve. In the discrete case, an arbitrary nonintersecting, closed $N$-sided curve shrinks in on itself, and its enclosed area vanishes in a finite time. We conjecture that the discrete curve tends to an equi-angle $N$-polygon as it shrinks.
\end{abstract}

Geometrical models which describe the motion of manifolds (curves and surfaces) in a higher dimensional space have been used successfully in various branches of science [12]. A simple example that has been studied extensively is the so-called curveshortening equation $[4,6,7]$, which describes the planar motion of a smooth curve that moves normal to itself with a speed proportional to its local curvature:

$$
\frac{d \mathbf{r}}{d t}=U \mathbf{n}, \quad U=\kappa,
$$

Here $\mathbf{r}$ is the position vector of a point on the curve, $\mathbf{n}$ is the inward-facing unit normal vector of the curve, and $\kappa$ is its curvature. Two important features of such motion are: (1) that every smooth closed curve shrinks to a point in a finite time; and (2) that the asymptotic shape of such a shrinking curve is always a circle.

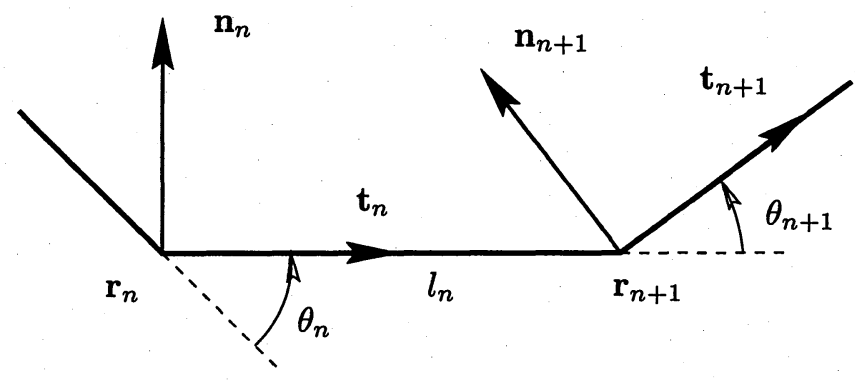

Figure 1. A discrete curve

The purpose of this paper is to formulate a discrete analogue of the curve-shortening equation, and to analyze its behavior. This is one of several discrete models of curveshortening that have been proposed $[2,3,8,9,13,14]$. We discuss some relations

Received December 18, 1995, revised July 25, 1996.

1991 Mathematics Subject Classification: 34A26, 73B30, 35K20.

Key words and phrases: the curve-shortening equation, discretization, geometric model. 
between these models at the end of this paper. We begin with a description of a discrete curve, (i.e., a piecewise linear curve) in a plane. The curve consists of $N$ points, connected by straight line-segments ("links"). The position vector of the $n$-th point is denoted by $\mathbf{r}_{n}$. By definition (Figure 1), we have

$$
\mathbf{r}_{n+1}=\mathbf{r}_{n}+l_{n} \mathbf{t}_{n}
$$

where $t_{n}$ is the unit "tangent" vector and $l_{n}$ is the length of the $n$-th link. The unit "normal" vector $\mathbf{n}_{n}$ is chosen to be a $90^{\circ}$ counter-clockwise rotation of $\mathbf{t}_{n}$ :

$$
\mathbf{n}_{n} \cdot \mathbf{t}_{n}=0 \text {. }
$$

Each pair of vectors $\left\{\mathbf{t}_{n}, \mathbf{n}_{n}\right\}$ forms an orthonormal basis for the plane. Thus there exists a set of angles $\left\{\theta_{n}\right\}$ (Figure 1) such that

$$
\left(\begin{array}{l}
\mathbf{t}_{n+1} \\
\mathbf{n}_{n+1}
\end{array}\right)=\left(\begin{array}{cc}
\cos \theta_{n+1} & \sin \theta_{n+1} \\
-\sin \theta_{n+1} & \cos \theta_{n+1}
\end{array}\right)\left(\begin{array}{l}
\mathbf{t}_{n} \\
\mathbf{n}_{n}
\end{array}\right) .
$$

Equation (3) is referred to as the discrete Serret-Frenet equation [5, 11].

The discrete Serret-Frenet equation (3) can be rewritten as

$$
\begin{aligned}
& \mathbf{t}_{n+1}=\mathbf{t}_{n}+\tan \frac{\theta_{n+1}}{2} \mathbf{n}_{n}+\tan \frac{\theta_{n+1}}{2} \mathbf{n}_{n+1}, \\
& \mathbf{n}_{n+1}=\mathbf{n}_{n}-\tan \frac{\theta_{n+1}}{2} \mathbf{t}_{n}-\tan \frac{\theta_{n+1}}{2} \mathbf{t}_{n+1} .
\end{aligned}
$$

It is interesting to compare (4) with the Ablowitz-Ladik system for a certain class of integrable differential-difference equations $[1,5]$ :

$$
\begin{aligned}
& v_{1, n+1}=z v_{1, n}+Q_{n}(t) v_{2, n}+S_{n} v_{2, n+1}, \\
& v_{2, n+1}=\frac{1}{z} v_{2, n}+R_{n}(t) v_{1, n}+T_{n}(t) v_{1, n+1} .
\end{aligned}
$$

We observe that the discrete Serret-Frenet equation is the Ablowitz-Ladik system at $z=1$ with $Q_{n}=S_{n}=-R_{n}=-T_{n}$. The corresponding statement for smooth curves is known [10].

We now consider a motion of a discrete curve. Let $t$ denote time. We express the velocity of the $n$-th point by

$$
\frac{d \mathbf{r}_{n}}{d t}=U_{n} \mathbf{n}_{n}+W_{n} \mathbf{t}_{n}
$$

Here $U_{n}$ and $W_{n}$ are the $\mathbf{n}_{n}$ and $\mathbf{t}_{n}$ components of the velocity, respectively. Explicit forms of $U_{n}$ and $W_{n}$ can be chosen to represent a variety of physical or geometrical phenomena. We require the consistency condition imposed on the dynamical variables,

$$
\frac{d}{d t} E=E \frac{d}{d t}
$$

where $E$ is the shift operator

$$
E f_{n}=f_{n+1} \text {. }
$$


Applying (6) to (3) and (5), we get

$$
\begin{gathered}
\frac{d}{d t}\left(\begin{array}{c}
\mathbf{t}_{n} \\
\mathbf{n}_{n}
\end{array}\right)=\left(\begin{array}{cc}
0 & \omega_{n} \\
-\omega_{n} & 0
\end{array}\right)\left(\begin{array}{l}
\mathbf{t}_{n} \\
\mathbf{n}_{n}
\end{array}\right), \\
\frac{d}{d t} l_{n}=\gamma_{n} \\
\frac{d}{d t} \theta_{n}=\Delta \frac{\omega_{n-1}}{l_{n-1}}
\end{gathered}
$$

where

$$
\left(\begin{array}{c}
\gamma_{n} \\
\omega_{n}
\end{array}\right)=\left(\begin{array}{cc}
\cos \theta_{n+1} & -\sin \theta_{n+1} \\
\sin \theta_{n+1} & \cos \theta_{n+1}
\end{array}\right)\left(\begin{array}{l}
W_{n+1} \\
U_{n+1}
\end{array}\right)-\left(\begin{array}{l}
W_{n} \\
U_{n}
\end{array}\right)
$$

and $\Delta$ is the difference operator

$$
\Delta f_{n}=(E-1) f_{n}=f_{n+1}-f_{n} .
$$

It is worth mentioning that all of the formulae for a smooth curve are recovered in the continuum limit

$$
l_{n} \rightarrow 0 \text { and } \theta_{n} \rightarrow 0 \text {. }
$$

In fact, in the limit (11), eqs. (2), (3), (7)-(9) become

$$
\begin{gathered}
\mathbf{t}=\frac{\partial \mathbf{r}}{\partial s} \\
\frac{\partial}{\partial s}\left(\begin{array}{l}
\mathbf{t} \\
\mathbf{n}
\end{array}\right)=\left(\begin{array}{cc}
0 & \kappa \\
-\kappa & 0
\end{array}\right)\left(\begin{array}{l}
\mathbf{t} \\
\mathbf{n}
\end{array}\right) \\
\frac{\partial}{\partial t}\left(\begin{array}{l}
\mathbf{t} \\
\mathbf{n}
\end{array}\right)=\left(\begin{array}{cc}
0 & \omega \\
-\omega & 0
\end{array}\right)\left(\begin{array}{l}
\mathbf{t} \\
\mathbf{n}
\end{array}\right) \\
\frac{\partial}{\partial t} \sqrt{g}=\sqrt{g} \gamma \\
\frac{\partial}{\partial t} \kappa=\frac{\partial}{\partial s} \omega
\end{gathered}
$$

where

$$
\left(\begin{array}{l}
\gamma \\
\omega
\end{array}\right)=\left[\frac{\partial}{\partial s}+\left(\begin{array}{cc}
0 & -\kappa \\
\kappa & 0
\end{array}\right)\right]\left(\begin{array}{l}
W \\
U
\end{array}\right)
$$

Here $g$ is the metric related to the length of the link, $l$, by $l=\sqrt{g} \sigma$ with $\sigma$ being a time-independent parameter.

The discussion so far is general. Next, we propose a specific model which leads to a curve-shortening equation. We assume that the velocity of the $n$-th particle is determined by

$$
\begin{gathered}
U_{n}=\frac{\theta_{n}}{l_{n}}, \\
W_{n}=\frac{\theta_{n}\left(l_{n}-l_{n-1} \cos \theta_{n}\right)}{l_{n} l_{n-1} \sin \theta_{n}} .
\end{gathered}
$$


See the Appendix for the derivation. In the continuum limit (11), (5) with (12) yields (1). Making use of (12) in (8) and (9), we obtain

$$
\begin{gathered}
\frac{d l_{n}}{d t}=-\frac{\theta_{n+1}}{l_{n+1} \sin \theta_{n+1}}-\frac{\theta_{n}}{l_{n-1} \sin \theta_{n}}+\frac{\theta_{n+1} \cos \theta_{n+1}}{l_{n} \sin \theta_{n+1}}+\frac{\theta_{n} \cos \theta_{n}}{l_{n} \sin \theta_{n}} \\
\frac{d \theta_{n}}{d t}=\frac{\theta_{n+1}-\theta_{n}}{l_{n}^{2}}-\frac{\theta_{n}-\theta_{n-1}}{l_{n-1}^{2}} .
\end{gathered}
$$

The model (12) has remarkable features. In what follows, we assume that the discrete curve is closed, and that it does not intersect itself.

Theorem 1. The area $S$ enclosed by the curve satisfies

$$
\frac{d S}{d t}=-2 \pi
$$

Proof. The area $S$ can be expressed as

$$
S=\frac{1}{2} \sum_{n} \mathbf{r}_{n} \times \mathbf{r}_{n+1} .
$$

Making use of (2) and (7), we obtain

$$
\frac{d S}{d t}=-\sum_{n} \theta_{n}=-2 \pi<0 .
$$

Comment 1. It follows that the area enclosed by the curve vanishes in a finite time, and that the time required is proportional to the initial area enclosed. The second equality in (17) is a special case of the Gauss-Bonnet theorem.

The usual (continuous) curve-shortening equation has property (15); the model (12) was chosen in order to maintain this property in the discrete version. This correspondence is discussed in detail in the Appendix.

Theorem 2. The total length $L=\sum l_{n}$ decreases,

$$
\frac{d L}{d t}=-\sum_{n}\left(\frac{1}{l_{n}}+\frac{1}{l_{n-1}}\right) \theta_{n} \tan \frac{\theta_{n}}{2}<0 .
$$

Proof. From the definition of $L$ and (8), it is easy to show (18). Note that $\left|\theta_{n}\right|<\pi$.

Theorem 3. A shrinking regular $N$-polygon is linearly stable to arbitrary small perturbations other than dilatation and rigid-body motion.

Proof. Let us introduce a new time variable $\tau$ by

$$
\frac{d t}{d \tau}=S, \quad \tau(t=0)=0 .
$$

The area $S$ can be expressed in two ways as

$$
S=S_{0}-2 \pi t=S_{0} \exp (-2 \pi \tau)
$$

where $S_{0}=S(t=0)$. 
Since the curve is closed, the closing conditions are

$$
\sum_{j=0}^{N-1} \theta_{j}=2 \pi, \quad \sum_{j=0}^{N-1} l_{j} \mathbf{t}_{j}=0 .
$$

For a regular $N$-polygon, we have

$$
\theta_{n}=\theta \equiv \frac{2 \pi}{N}, \quad l_{n}=l(t), \quad S(t)=\frac{1}{4} N l(t)^{2} \cot \frac{\theta}{2} .
$$

Equation (13) can be solved explicitly to yield

$$
l(t)=l(0) \sqrt{1-\frac{t}{t_{0}}}, \quad l(0)^{2}=4 \theta t_{0} \tan \frac{\theta}{2} .
$$

Now consider small deviations around the regular polygon. Expanding $\theta_{n}$ and $l_{n}$ as

$$
\theta_{n}=\theta+\delta \theta_{n}, \quad l_{n}=l+\delta l_{n}
$$

and inserting these into (13) and (14), we obtain

$$
\begin{aligned}
\frac{d}{d \tau} \delta \theta_{n}= & \frac{N}{4} \cot \frac{\theta}{2}\left(\delta \theta_{n+1}-2 \delta \theta_{n}+\delta \theta_{n-1}\right) \\
\frac{d}{d \tau} \delta l_{n}= & \frac{N}{8} \theta \csc ^{2} \frac{\theta}{2}\left(\delta l_{n+1}-2 \cos \theta \delta l_{n}+\delta l_{n-1}\right) \\
& -\frac{N}{4} l\left(1+\frac{\theta}{\sin \theta}\right)\left(\delta \theta_{n+1}+\delta \theta_{n}\right) .
\end{aligned}
$$

The discrete Fourier transformations,

$$
\tilde{\delta}_{k}=\sum_{n=0}^{N-1} \delta \theta_{n} \exp (-i k n \theta), \quad \widetilde{\delta l}_{k}=\sum_{n=0}^{N-1} \delta l_{n} \exp (-i k n \theta)
$$

recast these into

$$
\begin{aligned}
\frac{d}{d \tau} \widetilde{\delta \theta}_{k}= & -N \cot \frac{\theta}{2} \sin ^{2} \frac{k \theta}{2} \cdot \widetilde{\delta \theta}_{k} \\
\frac{d}{d \tau} \widetilde{\delta l}_{k}=- & \frac{N \theta}{4 \sin ^{2} \frac{\theta}{2}}(\cos \theta-\cos k \theta) \cdot \widetilde{\delta l}_{k} \\
& -\frac{N}{4} l\left(1+\frac{\theta}{\sin \theta}\right)(\exp (i k \theta)+1) \cdot \widetilde{\delta \theta}_{k}
\end{aligned}
$$

with the closing condition

$$
\widetilde{\delta \theta}_{0}=0, \quad \widetilde{\delta l}_{N-1}+\frac{i l}{1-\exp (i \theta)} \widetilde{\delta \theta}_{N-1}=0
$$

Note that $\widetilde{\delta l}_{1}=\widetilde{\delta l}_{N-1}^{*}$. Equations (27) can be solved easily. Their solutions show that all of the components go to zero as $\tau \rightarrow \infty$, except for $\widetilde{\delta l}_{0}$. The Fourier mode $\widetilde{\delta l}_{0}$ represents a dilatation.

Comment 2. A time variable $\tau$ defined by (19) turns out to be convenient also for later discussions.

Moreover, some exact results have been obtained. 
Theorem 4. An arbitrary triangle shrinks to a point in a finite time, and its limiting shape is a regular triangle.

Proof. The allowed region $\Omega$ for the angle variables is given by

$$
\Omega=\left\{\left(\theta_{0}, \theta_{1}, \theta_{2}\right) \mid \theta_{0}+\theta_{1}+\theta_{2}=2 \pi, \theta_{n}<\pi\right\} .
$$

Because the area $S$ can be expressed as

$$
S=\frac{1}{2} l_{2} l_{0} \sin \theta_{0}=\frac{1}{2} l_{0} l_{1} \sin \theta_{1}=\frac{1}{2} l_{1} l_{2} \sin \theta_{2},
$$

we have

$$
\begin{aligned}
& \frac{S}{l_{0}^{2}}=\frac{1}{2} \frac{\sin \theta_{0} \sin \theta_{1}}{\sin \theta_{2}} \\
& \frac{S}{l_{2}^{2}}=\frac{1}{2} \frac{\sin \theta_{2} \sin \theta_{0}}{\sin \theta_{1}} .
\end{aligned}
$$

Using these relations in (14), we obtain time evolution equations for the angles,

$$
\frac{d \theta_{0}}{d \tau}=\frac{1}{2} \sin \theta_{0} \sin \theta_{1} \sin \theta_{2}\left[\frac{\theta_{1}-\theta_{0}}{\sin ^{2} \theta_{2}}-\frac{\theta_{0}-\theta_{2}}{\sin ^{2} \theta_{1}}\right],
$$

and cyclic permutations. Let us introduce a function

$$
V=-\left(\pi-\theta_{0}\right)\left(\pi-\theta_{1}\right)\left(\pi-\theta_{2}\right) \text {. }
$$

The function $V$ is negative in $\Omega$, zero on $\partial \Omega$, and has a unique minimum at $P_{*}=$ $(2 \pi / 3,2 \pi / 3,2 \pi / 3)$. Its time derivative is given by

$$
\frac{d V}{d \tau}=-\frac{1}{2} \sin \theta_{0} \sin \theta_{1} \sin \theta_{2}\left[\frac{\left(\pi-\theta_{2}\right)\left(\theta_{1}-\theta_{0}\right)^{2}}{\sin ^{2} \theta_{2}}+\text { cyclic permutations }\right] .
$$

The right-hand side is negative on $\Omega \backslash\left\{P_{*}\right\}$ and zero at $P_{*}$. Thus $V$ can be considered as a Liapunov function, and therefore, $P_{*}$ is asymptotically stable.

Theorem 5. An arbitrary parallelogram shrinks to a point in a finite time, and its limiting shape is a rectangle.

Proof. Let $a=l_{0}, b=l_{1}, \theta=\theta_{0}$, and $\phi=\theta_{1}=\pi-\theta_{0}$. The evolution equations for the lengths and the angles are given by

$$
\begin{aligned}
& \frac{d a}{d \tau}=-\pi a+b(\phi-\theta) \cos \phi \\
& \frac{d b}{d \tau}=-\pi b+a(\phi-\theta) \cos \phi \\
& \frac{d \phi}{d \tau}=-(\phi-\theta) S\left(\frac{1}{a^{2}}+\frac{1}{b^{2}}\right), \\
& \frac{d \theta}{d \tau}=+(\phi-\theta) S\left(\frac{1}{a^{2}}+\frac{1}{b^{2}}\right)
\end{aligned}
$$

where $S=a b \sin \theta=a b \sin \phi=S_{0} \exp (-2 \pi \tau)$. Define $\Theta=(\phi-\theta) / 2, A=a \exp (\pi \tau)$, and $B=b \exp (\pi \tau)$. Since $0<\theta<\pi$, we have $-\pi / 2<\Theta<\pi / 2$. The evolution 
equations (33) can be recast into

$$
\begin{aligned}
& \frac{d A}{d \tau}=-2 B \Theta \sin \Theta \\
& \frac{d B}{d \tau}=-2 A \Theta \sin \Theta \\
& \frac{d \Theta}{d \tau}=-2 S_{0} \Theta\left(\frac{1}{A^{2}}+\frac{1}{B^{2}}\right) .
\end{aligned}
$$

Note that $\frac{d}{d \tau}\left(A^{2}-B^{2}\right)=0$. There are two cases.

(i) If $A=B$ initially, then the figure is initially equilateral, and it remains so. From (34c),

$$
\frac{d\left(\Theta^{2}\right)}{d \tau}=-\frac{4 S_{0} \Theta^{2}}{A^{2}} \leq-\frac{4 S_{0} \Theta^{2}}{A_{0}^{2}}
$$

where $A_{0}=A(\tau=0)$. So $\Theta \rightarrow 0$ as $\tau \rightarrow \infty$, and the equilateral parallelogram approaches a square as it shrinks to a point.

(ii) If $A \neq B$ initially, then we may assume without loss of generality that $A>B$, and that

$$
A=C \cosh \frac{u}{2}, \quad B=C \sinh \frac{u}{2}, \quad 0<u<\infty
$$

where $C$ is a positive constant. Now the evolution equations read

$$
\begin{gathered}
\frac{d u}{d \tau}=-4 \Theta \sin \Theta \\
\frac{d \Theta}{d \tau}=-\frac{8 S_{0}}{C^{2}} \Theta \frac{\cosh u}{\sinh ^{2} u} .
\end{gathered}
$$

Integrating these equations, we have

$$
\cos \Theta-\frac{2 S_{0}}{C^{2}} \frac{1}{\sinh u}=D=\text { constant. }
$$

Eliminating $u$ in (36b), we obtain

$$
\frac{d}{d \tau}\left(\Theta^{2}\right)=-\Theta^{2} f(\Theta)
$$

where

$$
f(\Theta)=\frac{4 C^{2}}{S_{0}}(\cos \Theta-D)^{2} \sqrt{1+\frac{4 S_{0}^{2}}{C^{4}(\cos \Theta-D)^{2}}} .
$$

Since $f(\Theta)>0$ for $u<\infty$, we have

$$
\frac{d}{d \tau}\left(\Theta^{2}\right)=-\Theta^{2} f(\Theta) \leq-\Theta^{2} f\left(\Theta_{0}\right)
$$

where $\Theta_{0}=\Theta(\tau=0)$. So $\Theta \rightarrow 0$ as $\tau \rightarrow \infty$, and the figure approaches a rectangle as it shrinks. Moreover, from (36a), $\frac{d u}{d \tau} \leq 0$, so $u \not \rightarrow \infty$ as $\tau \rightarrow \infty$. Thus $B / A=b / a \not \rightarrow 1$ as $\tau \rightarrow \infty$. In other words, the limiting figure is not a square.

Theorem 6. An arbitrary, pairwise symmetric (i.e., with opposite sides parallel and of equal length, as in Figure 2), equi-angle hexagon shrinks to a point in a finite time and its limiting shape is a regular hexagon. 


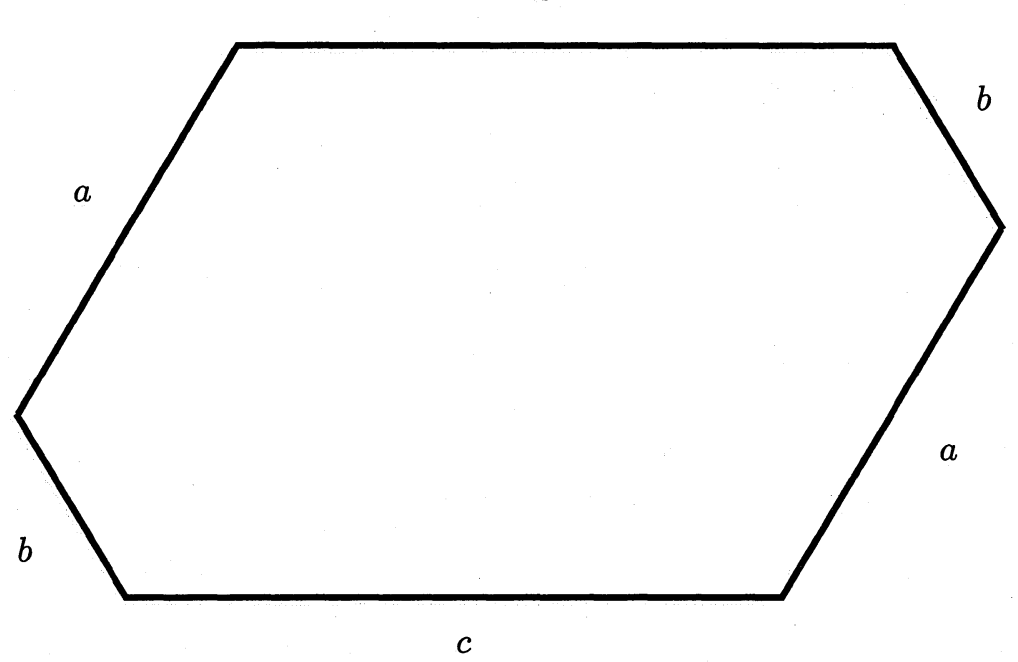

FIGURE 2. A pairwise symmetric, equi-angle hexagon

Proof. Let $a=l_{0}=l_{3}, b=l_{1}=l_{4}$, and $c=l_{2}=l_{5}$. Time evolution equations for these length variables are given by

$$
\frac{1}{\alpha} \frac{d a}{d t}=\frac{1}{a}-\frac{1}{b}-\frac{1}{c}
$$

and cyclic permutations, where $\alpha=2 \pi / 3 \sqrt{3}$. Define

$$
\begin{gathered}
L=a+b+c, \quad w=-\log L, \\
A=\frac{a}{L}, \quad B=\frac{b}{L}, \quad C=\frac{c}{L}, \\
Q=\frac{1}{A}+\frac{1}{B}+\frac{1}{C} .
\end{gathered}
$$

From (41), we get the following equations

$$
\begin{gathered}
\frac{d w}{d t}=\alpha \exp (2 w) Q \\
\frac{d A}{d w}=\frac{1}{Q}\left(\frac{1}{A}-\frac{1}{B}-\frac{1}{C}+A Q\right),
\end{gathered}
$$

and cyclic permutations. For $A, B$, and $C$, the allowed region $\Omega$ is given by

$$
\Omega=\{(A, B, C) \mid A+B+C=1, A, B, C>0\} .
$$

First let us show that $a, b, c \rightarrow 0$ simultaneously as $S \rightarrow 0$.

(1) Suppose $a \rightarrow 0$ and $b, c$ are finite as $S \rightarrow 0$. But (41) shows that if $0<a \ll b, c$, then $d a / d t>0$, so $\{a \rightarrow 0$ with $b, c$ finite $\}$ is impossible.

(2) Suppose $a, b \rightarrow 0$ and $c$ remains finite as $S \rightarrow 0$. Thus $d a / d t$ and $d b / d t$ should be negative for sufficiently small $S$. However, time evolution equations show that $(d a / d t)(d b / d t)<0$. Then this also never happens. 
(3) Thus we conclude that $a, b$, and $c$ simultaneously become zero as $S \rightarrow 0$. In particular, $w \rightarrow \infty$ as $S \rightarrow 0$.

Next we show that $a / b, a / c \rightarrow 1$ as $w \rightarrow \infty$. For this purpose, let us introduce a function,

$$
V=-A B C
$$

The function $V$ is zero on $\partial \Omega$, negative in $\Omega$, and has a unique minimum at $P_{*}=$ $(1 / 3,1 / 3,1 / 3)$. Its derivative $d V / d w$ is negative in $\Omega \backslash\left\{P_{*}\right\}$ and zero at $P_{*}$. Thus $V$ is a Liapunov function, and $P_{*}$ is asymptotically stable.

We conjecture that an arbitrary, pairwise symmetric, equi-angle $2 N$-polygon for $N>2$ shrinks to a point and its limiting shape is a regular one. The case $N=2$ is exceptional.

These properties ensure that the model (12) is appropriate for a discrete curveshortening equation. However, other models for discrete curve-shortening also have been proposed, as we now discuss. The first of these is due to Taylor, who set forth a theory for the motion of curves by crystalline curvature [2, 14]. A similar model was developed independently by Angenent and Gurtin [3], while Girão and Kohn [8, 9] establish the convergence of a numerical scheme (i.e., discrete in both space and time) for such motions. Their scheme can be compared to Roberts' [13] numerical scheme for the original curve-shortening equation.

Comparing our model (12) with Taylor's [2, 14], we note that in her model, linesegments translate in their normal directions, keeping their orientations. Her model is simpler than (12) because only $l_{n}$ varies in time, not $\theta_{n}$.

As we discuss in the Appendix, property (15) is an intrinsic property of the continuous curve-shortening equation, which is inherited by our model (12). Girão [8] shows that his model acquires this property in the continuum limit. A referee pointed out to us that Taylor's model exhibits this property (15) under some circumstances; whether that is always the case seems to be unknown.

\section{Appendix: A derivation of the model (12)}

In this Appendix we shall derive a discrete curve-shortening equation (5) with (12) from the continuous curve-shortening equation (1).

First of all, we note that the equation (1) is the one-dimensional reduction of a diffusion equation

$$
\frac{d \mathbf{r}}{d t}=\Delta \mathbf{r}
$$

where $\Delta=(\operatorname{det} g)^{-1 / 2} \partial_{\mu}(\operatorname{det} g)^{1 / 2} g^{\mu \nu} \partial_{\nu}$ is the Laplacian on the hypersurface $\mathbf{r}\left(u^{1}, \ldots, u^{k}\right)$ in $\mathbb{R}^{k+1}$ with respect to the induced metric $g$, and that the area $A$ enclosed by a simple closed curve driven by the flow (1) satisfies

$$
\frac{d A}{d t}=-\oint \kappa \sqrt{g} d u=-2 \pi \text {. }
$$


Equation (46) indicates that a natural discretization of (1) is

$$
\frac{d \mathbf{r}_{n}}{d t}=\frac{1}{g_{n}}\left(\mathbf{r}_{n+1}-2 \mathbf{r}_{n}+\mathbf{r}_{n-1}\right) \text {. }
$$

To determine $g_{n}$, we impose the condition (47) which also should hold for discrete curves since it represents a topological property of embedded curves. A little calculation shows that

$$
\frac{d A}{d t}=-\sum_{n} \frac{l_{n} l_{n-1} \sin \theta_{n}}{g_{n}}
$$

and the metric is identified as

$$
g_{n}=\frac{l_{n} l_{n-1} \sin \theta_{n}}{\theta_{n}}
$$

This choice of the metric gives a discrete curve-shortening equation (5) with (12). We remark that $g_{n}$ in (50) consists of geometric quantities associated only with the $n$-th vertex $\mathbf{r}_{n}$ (see Figure 1).

Acknowledgements. This work was supported in part by NSF grant DMS-9304390. This work also was supported in part by Monbusho International Scientific Research Program: Joint Research, 06044054, from the Ministry of Education, Culture, and Science, Japan.

\section{References}

1. M. J. Ablowitz and J. Ladik, Nonlinear differential-difference equations, J. Math. Phys. 16 (1975), 598-603.

2. F. Almgren and J. E. Taylor, Flat flow is motion by crystalline curvature for curves with crystalline energies, J. Diff. Geom. 42 (1995), 1-22.

3. S. Angenent and M. Gurtin, Multiphase thermomechanics with interfacial structure 2, Evolution of an isothermal interface, Arch Rat. Mech. Anal. 108 (1989), 323-391.

4. R. C. Brower, D. A. Kessler, J. Koplik, and H. Levine, Geometrical models of interface evolution, Phys. Rev. A 29 (1984), 1335-1342.

5. A. Doliwa and P. M. Santini, Integrable dynamics of a discrete curve and the Ablowitz-Ladik hierarchy, J. Math. Phys. 36 (1995), 1259-1273.

6. C. Epstein and M. Weinstein, A Stable Manifold Theorem for the Curve Shortening Equation, Comm. Pure Appl. Math. 40 (1987), 119-139.

7. M. Gage and R. S. Hamilton, The heat equation shrinking convex plane curves, J. Diff. Geom. 23 (1986), 69-96.

8. P. Girão, Convergence of a crystalline algorithm for the motion of a simple closed convex curve by weighted curvature, SIAM J. Numer. Anal. 32 (1995), 886-899.

9. P. Girão and R. Kohn, Convergence of a crystalline algorithm for the heat equation in one dimension and for the motion of a graph by weighted curvature, Numerische Mathematik 67 (1994), 41-70.

10. K. Nakayama, H. Segur, and M. Wadati, Integrability and the Motion of Curves, Phys. Rev. Lett. 69 (1992), 2603-2606.

11. M. Hisakado, K. Nakayama, and M. Wadati, Motion of Discrete Curves in the Plane, J. Phys. Soc. Jpn. 64 (1995), 2390-2393. 
12. P. Pelcé, Dynamics of Curved Fronts, Academic Press, 1988.

13. S. Roberts, A line element algorithm for curve flow problems in the plane, CMA Research Report 58, Australian Nat. Univ., 1989.

14. J. E. Taylor, Motion of curves by crystalline curvature including triple junctions and boundary points, Proceedings of Symposia in Pure Mathematics 54 (1993), 417-438.

Department of Mathematical Sciences, Faculty of Science, Shinshu University, Asahi 3-1-1, MATSUMOTO, NAGANO 390, JAPAN

Department of Applied Mathematics, University of Colorado, Boulder, Colorado 803090526, USA

Department of Physics, Graduate School of Science, University of Tokyo, Hongo 7-3-1, BUNKYO-KU, TOKYO 113, JAPAN 